\title{
Intrauterine levonorgestrel delivery with frameless fibrous delivery system: review of clinical experience
}

This article was published in the following Dove Press journal:

International Journal of Women's Health

21 January 2017

Number of times this article has been viewed

\author{
Dirk Wildemeersch ${ }^{1}$ \\ Amaury Andrade ${ }^{2}$ \\ Norman D Goldstuck ${ }^{3}$ \\ Thomas Hasskamp ${ }^{4}$ \\ Geert Jackers ${ }^{5}$
}

'Gynecological Outpatient Clinic and IUD Training Center, Ghent, Belgium: ${ }^{2}$ Centro de Biologia da Reprodução, Universidade Federal Juiz de Fora, Juiz de Fora, Brazil; ${ }^{3}$ Department of Obstetrics and Gynaecology, Faculty of Medicine and Health Sciences, Stellenbosch University and Tygerberg Hospital, Western Cape, South Africa; ${ }^{4}$ Klinik für Operativen Gynäkologie, GynMünster, Münster, Germany; ${ }^{5}$ Applied Controlled Release, Technology Park, Ghent (Zwijnaarde), Belgium
Correspondence: Dirk Wildemeersch Gynecological Outpatient Clinic and IUD Training Center, F Rooseveltlaan 43/44, 9000 Ghent, Belgium

Tel +3250600900

Emaildirk@wildemeersch.com
Abstract: The concept of using a frameless intrauterine device (IUD) instead of the conventional plastic framed IUD is not new. Frameless copper IUDs have been available since the late 1990s. They rely on an anchoring system to retain in the uterine cavity. The clinical experience with these IUDs suggests that frameless IUDs fit better as they are thin and, therefore, do not disturb or irritate the uterus. High tolerance and continuation rates have been achieved as complaints of pain are virtually nonexistent and the impact on menstrual blood loss is minimal. Conventional levonorgestrel-releasing intrauterine systems (LNG-IUSs) are very popular as they significantly reduce menstrual bleeding and provide highly effective contraception. However, continuation of use remains problematic, particularly in young users. Total or partial expulsion and displacement of the LNG-IUS also occur too often due to spatial incompatibility within a small uterine cavity, as strong uterine contractions originate, attempting to get rid of the bothersome IUD/ IUS. If not expelled, embedment ensues, often leading to chronic pain and early removal of the IUD/IUS. Several studies conducted recently have requested attention to the relationship between the LNG-IUS and the endometrial cavity. Some authors have proposed to measure the cavity width prior to inserting an IUD, as many uterine cavities are much smaller than the currently existing LNG-IUSs. A frameless fibrous drug delivery system fits, in principle, in all uterine cavities and may therefore be preferable to framed drug delivery systems. This review examines the clinical performance, acceptability, and potential of the frameless LNG-IUS (FibroPlant ${ }^{\circledR}$ ) when used for contraception, treatment of heavy menstrual bleeding, dysmenorrhea, and endometrial suppression in women using estrogen replacement therapy, endometrial hyperplasia, and other gynecological conditions. The review concludes that FibroPlant LNG-IUS offers unique advantages in reducing side effects.

Keywords: LNG-IUS, frameless, efficacy, safety, acceptability

\section{Introduction}

Synthetic fibers can be used as controlled delivery systems by a process where bioactive agents are combined with synthetic polymers and extruded to form filaments of various lengths and diameters. The process is continuous, resulting in precision drug delivery systems capable of releasing active agents at specific rates for extended periods of times. ${ }^{1}$ These controlled-release fibrous systems are used extensively in the medical field as implants, medical devices, and in oral delivery systems. The choice of polymer(s) used and the rate of release are dependent on the particular bioactive agent employed and the design of the fibrous delivery system. Many different polymers can be extruded into fibers. Candidate polymers that can be used for fibrous delivery systems include both nonbiodegradable and biodegradable polymers. In the 1980s, 
a nondegradable polymer, poly-ethylene-co-vinyl acetate (pEVA), was developed and evaluated for application in various medical products. Through the use of select polymers, such as pEVA, both zero-order (constant) and first-order (proportional) rates of release can be obtained, with some systems or combination of polymers yielding complex release rates that can be a combination of the two. Thus, complex pseudo-constant rates of release may be obtained from some fibrous devices. Fibrous delivery systems can also be processed to release different active pharmaceutical ingredients alone or in combination.

The area of contraception has greatly benefited from the advances made in controlled drug delivery. The most successful controlled drug delivery system to date is the family of implantable female contraceptive devices developed by the Population Council in the mid-1970s. The development of Norplant ${ }^{\circledR}$ (Wyeth Pharmaceuticals, Dallas, TX, USA) and Mirena ${ }^{\circledR}$ (Bayer HealthCare, Berlin, Germany) represented the first of a series of contraceptive delivery devices for subdermal and intrauterine use. ${ }^{2,3}$ They are capable of delivering levonorgestrel (LNG), or other hormones, at efficacious levels from the time of implantation or insertion up to a period of 5 years or longer.

We have optimized and tested LNG-releasing EVA-based fibers since the late 1990s for contraception and the treatment of gynecological conditions such as the treatment of heavy menstrual bleeding (HMB). These fibrous delivery systems can be designed to typically release $10 \mu \mathrm{g}$ to $20 \mu \mathrm{g}$ of LNG/ day and have maximal efficacy for at least 5 years, with a total lifespan of up to 10 years. These systems as well as other intrauterine drug-releasing devices have completely revolutionized birth control by offering a convenient, fail-safe way to prevent pregnancy requiring minimal patient intervention.

Various gynecological conditions exist in women, which affect not only a women's reproductive function but also her overall quality of life. Many are accompanied by the presence of menorrhagia, dysmenorrhea, and dyspareunia. Abnormal uterine bleeding (AUB) is the most frequent gynecological complaint expressed by women with the incidence of complaints increasing as a woman approaches menopause. Heavy or excessive menstrual bleeding unfortunately occurs quite frequently. The prevalence has been reported to be between $9 \%$ and $28 \%$ in women aged 16 to 45 years and increases with age. ${ }^{4} \mathrm{Up}$ to $30 \%$ of patients referred for gynecological treatment are for menorrhagia, and half of these women have a hysterectomy within 5 years if conservative interventions (eg, contraceptive pills, progestogens, fibrinolytic inhibitors, and prostaglandin inhibitors) fail. ${ }^{5}$ It was found that more than one-third of the women undergoing a hysterectomy for menorrhagia have normal uteri with no underlying pathology. ${ }^{6}$

Fibroids (fibromyoma and leiomyoma) are often associated with HMB. Uterine fibroids are a prevalent condition, with a cumulative incidence of nearly $70 \%$ in White women and greater than $80 \%$ in black women by the age of 50 years. $^{7}$

Endometriosis is another frequent, debilitating, and costly condition in women characterized by the presence of endometrial tissue outside the uterus. ${ }^{8}$ Endometriosis typically occurs on the surface of organs in the abdominopelvic cavity and rectovaginal septum, or in the ovary, and is a chronic painful condition, particularly if the lesions are deep and infiltrating. Endometriosis affects almost 10\% to $20 \%$ of women of reproductive age, while $70 \%$ to $90 \%$ of women with chronic pelvic pain (CPP), dysmenorrhea, dyspareunia, infertility, and menstrual disturbances also harbor endometriotic lesions.

Adenomyosis is a specific form of endometriosis characterized by the presence of ectopic endometrial glands in the uterine wall, associated with hyperplasia of the surrounding muscles. Adenomyosis is present in at least 1\% of the female population. ${ }^{9}$ It is not uncommon for women with adenomyosis and endometriosis to present with HMB associated with adenomyosis.

Although less frequent than conditions of menorrhagia or endometriosis, endometrial hyperplasia is another condition where women may experience AUB. Endometrial hyperplasia is considered a precursor of cancer of the endometrium. Most women presenting with endometrial hyperplasia have benign non-atypical hyperplasia. However, women presenting with atypical hyperplasia have a risk of developing endometrial carcinoma that is estimated to be between $27 \%$ and $30 \% .{ }^{10}$ Most often, they consult with complaints of AUB.

Historically, hysterectomy is the most commonly used procedure to treat $\mathrm{HMB}$, fibroids, and precancerous lesion or early cancer of the endometrium although medical treatment is preferable, at least for some conditions. Mild cases of endometriosis can be managed medically with contraceptive steroids and nonsteroidal anti-inflammatory agents. Laparoscopic surgical removal of endometriosis is an effective first-line approach for treating pain related to endometriosis. ${ }^{11}$ Use of various uterine ablation procedures to alleviate the condition has been growing. However, these techniques fail to treat distal adhesions or early-stage microscopic implants that 
may be present outside of the uterus. More importantly, future fertility is compromised. Surgery provides immediate pain relief to many women, but symptoms recur in up to $75 \%$ of cases within 2 years. ${ }^{12}$ Unfortunately, removal of a women's uterus is the ultimate last resort for many patients.

Current medical practice relies on strategies that delay as long as possible the need for surgical interventions and/or hysterectomy to treat many of these cases. The development of new, long-acting, therapeutic options, such as the levonorgestrel-releasing intrauterine system (LNG-IUS), have been shown to play a significant role in alleviating many of the symptoms women with these conditions exhibit. These systems have been investigated for the local or loco-regional treatment of the gynecological conditions described earlier. LNG-IUS has been tested with success in women with HMB, endometriosis, and adenomyosis. HMB associated with fibromyomas can often be treated effectively with LNG-IUS. As a LNG-IUS is also a highly effective contraceptive, it can be considered a multipurpose treatment modality providing women relief for one or more gynecological conditions while offering contraception simultaneously.

Various intrauterine drug delivery systems are available worldwide with the major ones being Mirena and Liletta ${ }^{\circledR}$ (Actavis Pharma, Parsippany, NJ, USA). Although having marked effectiveness, patient acceptance and continuation rates are many times less than ideal. Numerous clinical studies of Mirena in women seeking contraception have reported continuation rates of only $50 \%$ or lower over its clinical lifespan. Complaints of cramping, pain, dysmenorrhea, and spotting contribute to its less than optimal patient acceptance. Alternate designs including smaller devices, such as Jaydess/Skyla (Bayer HealthCare), and/or altering retention mechanisms have been developed in an effort to improve overall patient acceptance and tolerability.

This review focuses on the performance and acceptability of two frameless LNG-IUSs releasing $20 \mu \mathrm{g}$ and $14 \mu \mathrm{g}$ of LNG/day, respectively, when used for contraception, for the treatment of HMB, for excessive bleeding associated with uterine fibroids, for the treatment of precancerous changes of the uterus, for endometrial suppression in women using estrogen replacement therapy, and for the treatment of primary or secondary dysmenorrhea.

\section{Materials and methods}

\section{Description of the FibroPlant ${ }^{\circledR}$ LNG-IUS}

FibroPlant ${ }^{\circledR}$ LNG-IUS (APCOR, Ghent, Belgium) is a multicomponent system consisting of a non-resorbable thread whose proximal end is provided with a single knot. Attached thereto is a $3.0 \mathrm{~cm}$ or $3.5 \mathrm{~cm}$ long and $\sim 1.2 \mathrm{~mm}$ or $1.6 \mathrm{~mm}$ wide fibrous delivery system, releasing approximately $14 \mu \mathrm{g}$ and $20 \mu \mathrm{g}$ of LNG/day, respectively. The low-dose system is effective for 3 years and the high-dose system for 5 years. The fiber is fixed to the anchoring thread by means of a metal clip $1 \mathrm{~cm}$ from the anchoring knot. The anchoring knot is implanted into the myometrium of the uterine fundus using an insertion instrument, permanently securing the implant in the uterine cavity (Figure 1). A stainless steel marker is added below the anchoring knot, allowing for proper location of the IUS in the uterine cavity at insertion and on follow-up (Figure 2).

\section{Insertion procedure}

Insertion of the anchor is accomplished by pushing the inserter forward over a controlled distance of $10 \mathrm{~mm}$. Insertion procedure is identical with that of the GyneFix ${ }^{\circledR}$ copper IUD (http://www.wildemeersch.com). The anchor point and the drug delivery fiber are clearly visible on ultrasound. This is important as the provider can check if proper insertion has been realized (Figure 2). It is also recommended to measure the distance between the surface of the uterus and the marker point (S-A distance) allowing to compare this distance with the distance measured at follow-up.

\section{Contraceptive studies}

A total of 304 women were included in the contraceptive study. Most women were parous; only $14 \%$ of the participants were nulliparous women. Women were screened for their clinical suitability for intrauterine device (IUD) insertion and compliance with the World Health Organization (WHO) eligibility criteria. ${ }^{13}$ The experimental use of the Fibroplant LNG-IUS

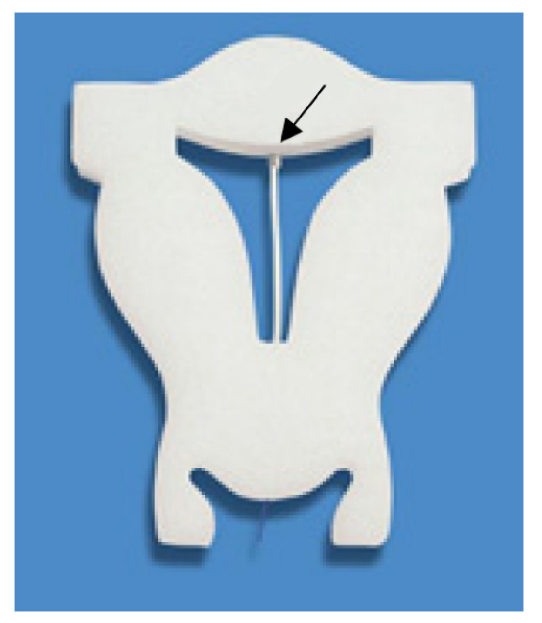

Figure I FibroPlant ${ }^{\circledR}$ LNG-IUS releasing $14 \mu \mathrm{g}$ or $20 \mu \mathrm{g}$ of LNG/day has a diameter of $1.2 \mathrm{~mm}$ and $1.6 \mathrm{~mm}$, respectively. The stainless steel clip securing the fiber to the anchoring thread is shown (arrow).

Abbreviation: LNG-IUS, levonorgestrel-releasing intrauterine system. 

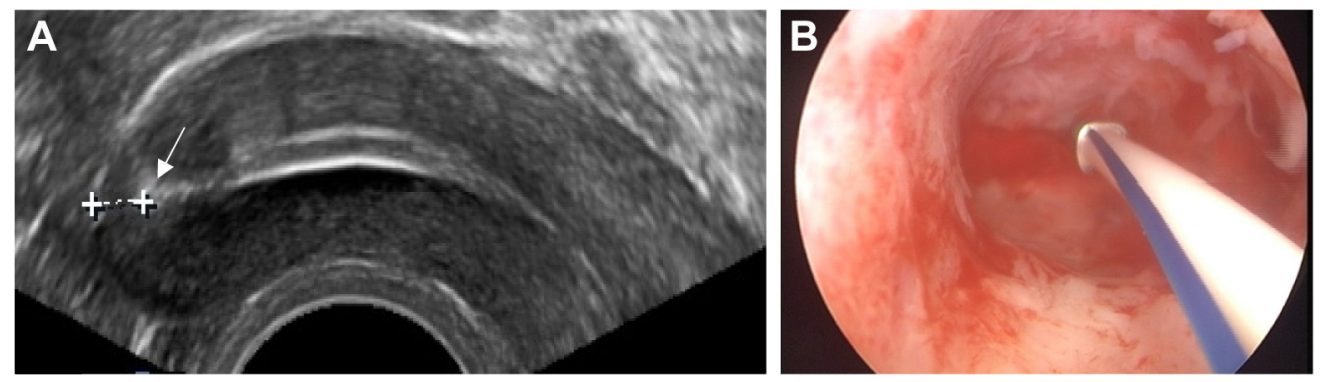

Figure 2 (A) 2D ultrasound of FibroPlant ${ }^{\circledR}$ LNG-IUS in situ. The stainless steel marker or anchor point is shown (arrow). The distance of the anchor point from the serosa is $3.3 \mathrm{~mm}$ in this case. The fibrous delivery systems are also clearly visible; (B) hysteroscopic picture of FibroPlant LNG-IUS, implanted in the fundus of the uterus. Abbreviations: LNG-IUS, levonorgestrel-releasing intrauterine system; 2D, two dimensional.

was approved by the Ethics Committee of the University of Ghent, Belgium. All women admitted in the study signed a written informed consent form. Before insertion of the LNGIUS, a medical history was taken and a pelvic examination was carried out. Care was taken to rule out any pelvic infection and, if judged necessary, clinical signs of sexually transmitted diseases (STDs) were ruled out. In case of doubt, bacteriological tests for chlamydia and gonorrhea were done. In each patient, a transvaginal ultrasound (TVU) examination was performed to evaluate if the uterus appeared normal. Following insertion of a FibroPlant, releasing $20 \mu \mathrm{g} /$ day of LNG, a new TVU was conducted to check the position of the IUS and to measure the S-A distance. Follow-up examinations were conducted at 1, 6, 12 months and yearly thereafter up to 60 months following insertion, and each time the position of the LNG-IUS was checked. Women were asked about bleeding patterns and adverse effects by direct questioning.

All data that were generated during the study were transferred to the data-coordinating center at the Department of Medical Informatics and Statistics, University Hospital, Gent, Belgium. The rates of discontinuation for individual reasons and groups of reasons were analyzed using the S-PLUS statistical software package (Mathsoft Corp, Cary, $\mathrm{NC}$, USA), ${ }^{14}$ and the cumulative discontinuation rates were computed using survival analysis methods. ${ }^{15,16}$

A smaller, multicenter contraceptive study using the frameless copper and the frameless LNG-IUD was conducted in 154 young nulliparous women of whom 50 were fitted with the FibroPlant releasing $20 \mu \mathrm{g}$ of LNG/day. All participants in the study were screened as to their clinical suitability for IUD insertion and compliance with the WHO eligibility criteria. ${ }^{13}$ Follow-up was done according to routine criteria.

\section{Menstrual blood loss studies}

The FibroPlant system, releasing $20 \mu \mathrm{g}$ of LNG/day, was used in the first menstrual blood loss (MBL) study and the
$14 \mu \mathrm{g} /$ day releasing LNG-IUS in the quantitative MBL study. FibroPlant use was allowed by the respective Ethics Committees and informed consent was obtained from the participants. Prior to insertion of the FibroPlant system, women were admitted to the studies in compliance with the WHO eligibility criteria. ${ }^{13}$

The visual assessment technique as described by Janssen et $\mathrm{al}^{17}$ was used in the first MBL study conducted in 32 Belgian women complaining of HMB in association or not with prior IUD use. Women with dysfunctional uterine bleeding, without gross abnormalities of the uterus (four women had small intramural fibroids) or any other pathology, were included. A menstrual score of $\geq 185$ was used as cutoff point for menorrhagia, as this score has a predictive value of $85 \%$ to be consistent with menorrhagia. Amenorrhea was defined as absence of MBL during a period of 90 days. Follow-up lasted for 12 months. The menstrual score and ferritin levels were assessed during this time interval.

In the second MBL study conducted in 40 fertile Brazilian women having normal or dysfunctional uterine bleeding $(\geq 80 \mathrm{~mL}$ ), the quantitative alkaline hematin technique as described by Newton et al was used. ${ }^{18}$ Blood loss was measured before insertion of the LNG-IUS (baseline controls) and after 3, 6, 12, and 24 months of insertion. The trial nurse instructed women to carefully collect their menstrual tampons and bring them to the laboratory in opaque plastic bags as soon as bleeding ended, as described previously. ${ }^{19}$ Serum ferritin level was also measured at the same time intervals as mentioned earlier.

\section{Endometrial suppression in perimenopausal and postmenopausal women using estrogen replacement therapy}

One hundred and fifty perimenopausal and postmenopausal women who complained of climacteric symptoms and/or 
menstrual problems were enrolled in this study. The study was approved by the Ethics Committee of the University of Ghent, Belgium, and written informed consent was obtained from all women admitted to the study. All women were screened for their clinical suitability for insertion of an IUS and compliance with the WHO eligibility criteria. ${ }^{13}$ Before the placement of the LNG-IUS, women were examined by TVU to ascertain normality of the uterus and pelvic organs, and the patient was checked for clinical signs of STDs. Since women included in the study were at low risk for STDs, no routine chlamydia tests were done. A wet vaginal smear was obtained to evaluate the hormonal status of the patient. If an atrophic status of the uterus was found, estrogen therapy (ET) was started immediately and insertion of the FibroPlant system, releasing $14 \mu \mathrm{g}$ of $\mathrm{LNG} /$ day, was postponed until the uterus was sufficiently primed and a wet smear showed the absence of parabasal cells (usually 1 month later). Most insertions were performed without local or paracervical anesthesia. Following insertion, a TVU was performed to identify the precise position of the LNG-IUS in the uterus. Estrogen was administered as either a percutaneous estrogencontaining gel (Oestrogel ${ }^{\circledR}$; Besins International, Montrouge, France) in a dosage of 0.75 to $1.5 \mathrm{mg}$ /day or a transdermal matrix system $\left(\right.$ Systen $^{\circledR}$; Janssen-Cilag, New Brunswick, NJ, USA) in a dosage of $50 \mu \mathrm{g} /$ day.

An endometrium biopsy was taken with a suction curette (Probet $^{\circledR}$; Gynétics Medical Products, Lommel, Belgium) prior to device replacement in 100 women to assess the impact of the regimen on the endometrium. The samples were drawn from all parts of the uterus in order to obtain a representative picture. The biopsies were placed in $4 \%$ phosphate-buffered formaldehyde immediately upon collection and stained with hematoxylin and eosin for examination. Two pathologists examined the pathology slides according to the diagnostic categories of Hendrickson and Kempson. ${ }^{20}$

\section{Endometrial suppression in women with non-atypical and atypical endometrial hyperplasia}

Each of the 20 women in the study, of whom 8 were diagnosed with atypical hyperplasia, received a LNG-IUS, releasing $14 \mu \mathrm{g}$ of LNG/day. The frameless IUS was used until expiry after 3 years and then replaced with a T-shaped LNG-IUS (Femilis ${ }^{\circledR}$; APCOR), releasing $20 \mu \mathrm{g}$ of $\mathrm{LNG} /$ day. At the time of study analysis, 13 patients were using the T-shaped LNG-IUS and 7 using the frameless LNG-IUS. Women with AUB and endometrial hyperplasia, confirmed by pipelle biopsy or by curettage, were fitted with the LNG-IUS for local treatment of the condition. Written informed consent was obtained from each volunteer and the use of the LNGIUS was approved by the Ethics Committee of the Ghent University Hospital. Women were screened pretreatment according to their clinical suitability for IUS insertion and compliance with the WHO eligibility criteria. ${ }^{10}$ Following insertion, the endometrium was monitored during treatment by TVU and by endometrial pipelle biopsy or dilatation and curettage (D\&C) (after prior removal of the LNG-IUS).

The histopathological diagnosis (Kurman classification ${ }^{21}$ ) was non-atypical (simple) hyperplasia in 12 women and atypical hyperplasia in 8 women (adenomatous hyperplasia with atypia in 3 among them).

\section{Study in patients with primary and secondary dysmenorrhea}

The 21 patients included in this study complained of painful periods, often requiring to stay home from school or work and sometimes associated with heavy menstrual periods. The history included age at menarche; menstrual frequency; length of period; estimate of the menstrual flow; associated symptoms (eg, nausea, vomiting, or other symptoms); severity of pain and its relationship with the menstrual cycle (women who had to stay at home were considered to have severe dysmenorrhea); impact on physical and social activity; and progression of symptom severity. All women complied with the WHO eligibility criteria for IUD insertion. ${ }^{13}$ Women were examined to exclude pelvic pathology and clinical signs of STDs. Routine chlamydia tests were not carried out when it was considered that women were at low risk for STDs. TVU was performed to assess abnormalities of the genital organs or any other pathology in the pelvis. Women with fibroids were also evaluated by magnetic resonance imaging (MRI). Eighteen insertions were performed with FibroPlant releasing $14 \mu \mathrm{g}$ of LNG/day and three with FibroPlant releasing $20 \mu \mathrm{g}$ of LNG/day. Seven insertions were done in nulliparous women. Fifteen women complained of HMB. Three women had significant fibromyomas and three were suspected to have adenomyosis. Written informed consent was obtained from patients and the Ethics Committee approved the use of FibroPlant LNG-IUS in the study.

\section{In vivo release rate and duration of action of FibroPlant LNG-IUS}

The LNG content was analyzed in 15 patients following removal of the LNG-IUS. Seven FibroPlant LNG-IUSs, releasing $14 \mu \mathrm{g}$ of LNG/day, were analyzed by high-performance liquid 
chromatography coupled to ultraviolet detection following preparation of the test solution for the determination of LNG content in the fibrous delivery systems after Soxhlet extraction, to calculate the duration of release (Laboratory of Analytical Pharmaceutical Chemistry, University of Liège, Liège, Belgium). The LNG fibers were in situ from 12 to 46 months. The LNG content at the beginning was $13 \mathrm{mg}$ and the LNG-IUS was developed to last for 3 years.

Eight FibroPlant LNG-IUSs, releasing $20 \mu \mathrm{g}$ of LNG/day, were also analyzed using the same procedure. All fibers were in situ for 60 months. The LNG content at the beginning was $\sim 30 \mathrm{mg}$ and the LNG-IUS was developed to remain in situ for 5 years.

\section{Results}

\section{Contraceptive study}

Among a total of 304 women with a mean age of 34.7 (range 15-48) years, including 14\% nulliparous women and 11,299 woman-months of use with follow-up up to 5 years, one pregnancy occurred after unnoticed expulsion of the LNG-IUS. There were two total expulsions of the frameless LNG-IUS. In addition, two uterine perforations occurred at insertion, one during the physician's learning period with the new insertion technique following difficult removal of a displaced Mirena LNG-IUS, conducted under general anesthesia. The second perforation was probably caused by inadvertent excessive pressure application on the fundal wall during insertion. The malpositioned devices were removed by laparoscopy.

During the study period, 24 removals were done for abnormal bleeding and 12 for pain complaints. The majority of the latter were unrelated to the use of the LNG-IUS (eg, suspicion of pelvic inflammatory disease [PID], CPP, ovarian cyst, presence of pelvic kidney in one woman, personality disorder). In addition, seven removals of the LNG-IUS were done for "other medical reasons", of which four were for mood disturbances and three for partial displacement (ie, suboptimal anchoring of the LNG-IUS). The cumulative total use-related discontinuation rate was 23.6/100 women at 5 years. Sixteen women requested removal of the LNG-IUS with wish to become pregnant. Two among them could not be traced but all 14 remaining patients became pregnant, with a removal-conception interval of 4.5 to 122 weeks. Eighty-five percent of women became pregnant within 1 year after removal of the LNG-IUS. The FibroPlant LNG-IUS was well accepted by the large majority of nulliparous as well as by parous women. The study was well followed up and among a total of 304 women, only 7 could not be contacted, despite numerous efforts. ${ }^{22}$
Of the 50 young women in the second contraceptive study, 22 were adolescents. The mean duration of IUS use was 37.9 (range 2-85) months for the LNG-IUS. The continuation rate at 36 months was $95.7 \%$. There was one removal for planned pregnancy; one expulsion occurred and two removals were done for hormonal side effects. The study concluded that due to the optimal relationship with the uterine cavity; the FibroPlant LNG-IUS is well accepted, resulting in high continuation rates. ${ }^{23}$

\section{Menstrual blood loss studies}

All heavy menstrual bleeders in the first (Belgian) study using the pictorial bleeding assessment chart scoring system reported a significant reduction in MBL, which started from the first menstrual period following insertion of the IUS. Bleeding reduced further over the next months until the sixth month. No amenorrhea, defined as absence of bleeding during a 90-day period, occurred. The mean bleeding score before treatment was 338 (range 185-740) in the group with no prior IUD use and 368 (range 185-890) in the group with prior IUD use. During treatment, the menstrual score diminished to a mean score of 70 (range 5-210) in the "no prior IUD use" group and to a mean of 52 (range 3-150) in the "prior IUD use" group after 1 to 23 months of follow-up. This result is highly statistically significant $(P<0.001)$. There were no statistical differences in bleeding scores before and during treatment between the two groups of women with or without prior copper IUD use. The four women who had small intramural fibroids responded well to the treatment. Women with the highest score prior to the treatment also had the highest scores during treatment follow-up. ${ }^{24}$

The quantitative MBL study, conducted in 40 Brazilian women with normal menstrual bleeding and HMB, reported a strong reduction in MBL from a mean baseline menstrual volume of $29.7 \pm 2.2 \mathrm{~mL}$ to a mean volume of $1.5 \pm 2.8 \mathrm{~mL}$ after 24 months. The results in women with normal menstrual bleeding at baseline and those with HMB at baseline, due to hormonal disturbance, were not significantly different. During the course of the treatment, ferritin values increased from a mean baseline value of $31.1 \pm 3.2 \mathrm{ng} / \mathrm{mL}$ at baseline controls to a mean level of $72.5 \pm 2.1 \mathrm{ng} / \mathrm{mL}$ after 24 months of LNG-IUS use. The differences in menstrual volume and ferritin levels at baseline, compared to the menstrual volumes and ferritin levels at 12 and 24 months, respectively, were highly significant $(P<0.0005)$. It is remarkable that $80 \%$ of women reported amenorrhea after 24 months of use. This was also seen in other MBL studies conducted in Brazil, while the amenorrhea rates in the Belgian women were much lower, and were attributed to 
genetic factors. Reduction of bleeding was similar between the heavy bleeders and the normal bleeders, and the heavy bleeders had comparable MBL to the normal bleeders 3 months after insertion, and by 24 months post-insertion, their ferritin levels were comparable to those of the normal bleeders. ${ }^{25}$

\section{Study in perimenopausal and postmenopausal women using estrogen replacement therapy}

FibroPlant LNG-IUS was inserted in 150 perimenopausal and postmenopausal women. Ninety-four insertions were done in perimenopausal and 56 in postmenopausal women with age between 33 and 78 years, respectively. Insertion was difficult in two postmenopausal women due to cervical stenosis, necessitating sounding and dilatation of the cervical canal. In four perimenopausal women, fibroids were present, classified as single or multiple intramural and subserosal fibroids with no evidence of submucosal fibroids.

Of the total group of 150 women, there were $5(3.3 \%)$ removals for medical reasons. One removal was done for abnormal bleeding, one for pain complaints, one for "suspicion" of pelvic infection by a colleague, one prior to surgery for a paraovarian tumor, and one for weight gain. One woman was released from follow-up due to noncompliance with the study requirements. There were no expulsions. All perimenopausal women developed scanty menstrual bleeding or no bleeding at all $(\sim 30 \%)$. The total number of women continuing with the method at 12 months was 142 (94.6\%). The average number of months that the device was in situ was 40 (range 4-70). Only two (1.3\%) women were lost from the final analysis. The study, with total number of womanmonths of use of 6063, was well followed up.

The endometrial biopsies taken from 101 postmenopausal women prior to FibroPlant replacement, after an average period of use of the method of 40 months (range 25-50), showed predominantly inactive endometrium. There were no specimens showing signs of proliferation. Slight scanty and infrequent bloody discharge requiring no protection, or a small panty liner, occurred in some women during the use of the regimen but was usually observed for a short period of time, except in a minority of women. However, only one woman requested removal of the LNG-IUS for this reason. No serious adverse effects were observed (eg, PID and perforation). ${ }^{26}$

\section{Study in patients with endometrial hyperplasia}

The average age of the 20 patients included in the study was 54 (range 41-67) years, and the average duration of use of the LNG-IUS was 32 (range 14-90) months. All the patients were evaluated by TVU, and it was found that all of them developed a thin endometrium ( $\leq 4 \mathrm{~mm}$ in thickness). One patient with an endometrial polyp of $20 \mathrm{~mm}$ in diameter was allowed in the study as she refused surgical removal. Simple hyperplasia was found in this patient. The polyp diminished gradually in size to $5 \mathrm{~mm}$ at the last follow-up examination, 53 months after insertion of the LNG-IUS. All women presenting with atypical endometrial hyperplasia at the start of the study showed expression of progesterone receptor (PR) in the epithelial cells, but the percentage declined significantly during treatment. Not one histological specimen showed progression of disease. Profound endometrial suppression with glandular atrophy and/or stromal decidualization was found in all women. All women were recommended to continue the use of the method to prevent recurrence. ${ }^{27}$

\section{Study in patients with primary and secondary dysmenorrhea}

The frameless FibroPlant LNG-IUS was the only method used to alleviate the menstrual pain complaints in the 21 patients with primary and secondary dysmenorrhea in this study. Among these women, 12 were categorized as having primary dysmenorrhea, as no organic cause for the painful menstrual periods could be found. In three women, an anomaly of the uterus was found. Three women had significant multiple intramural or subserosal fibroids, as confirmed by sonography and MRI. Two other women had small fibroids and four had enlarged uteri without other apparent uterine anomalies, of whom three were suspected to have uterine adenomyosis. All women were followed up after 1, 3, 6, and 12 months. On questioning, all women reported significant reduction of menstrual bleeding and much reduced pain or no pain at all. One woman with significant fibroids reported much reduced bleeding, but this was not as pronounced as in the other women in the study. Further follow-up up to 2 years revealed persistent positive results with absence of pain and much reduced bleeding. ${ }^{28}$

\section{In vivo release rate and duration of action of the FibroPlant LNG-IUS}

The estimated duration of action of the FibroPlant LNG-IUS, releasing $14 \mu \mathrm{g}$ of LNG/day, calculated by extrapolation is estimated to be between 5 and 7 years given the zero-order release characteristics of the fibers. The daily release rate over 36 months was $\sim 6 \mu \mathrm{g} /$ day. The results of the FibroPlant LNG-IUS, releasing $20 \mu \mathrm{g}$ of LNG/day, indicated the duration of action of more than 8 years with a daily release rate of $\sim 10 \mu \mathrm{g} /$ day over 60 months (APCOR, data on file). 


\section{Discussion}

FibroPlant LNG-IUS acts profoundly on the morphology and the function of the endometrium like other types of LNGIUS. Both the $14 \mu \mathrm{g}$ and the $20 \mu \mathrm{g}$ releasing LNG-IUSs have an undistinguishable impact on the endometrium. ${ }^{29}$ The strong impact on the endometrium explains the high contraceptive and therapeutic efficacy of the LNG-IUS. The non-contraceptive benefits of a LNG-IUS for treatment and prevention are increasingly recognized and it is hardly possible nowadays to think of practicing gynecology without having the possibility to use a LNG-IUS for contraception and/or treatment of many gynecological conditions for which the LNG-IUS brings relief without having to rely on surgical intervention.

In contrast with other framed LNG-IUS, the frameless FibroPlant LNG-IUS has unique design characteristics. It could be regarded as the ultimate LNG-releasing IUS in many ways, as the thin fiber will be suitable for any uterine cavity, small and large, even if it is deformed by the presence of fibromyomas. As FibroPlant has no plastic frame, the fiber requires anchoring to prevent its expulsion. Anchoring a foreign body in the uterus is a new technique, hence the requirement for thorough physician training. Supervised training in a model is important to become familiar with the anchoring technique of insertion of the frameless LNG-IUS not only to boost physician confidence but also to minimize improper insertions and possible early-stage expulsions. After training, it typically requires only three/four live insertions for a physician to develop competency in the procedure. The anchoring mechanism for FibroPlant is identical to that used in the copper-releasing IUD, GyneFix, which is commercially available in Europe.

Assessing uterine fundal thickness by ultrasound will help physicians, unfamiliar with the procedure, to gain experience in providing a safe and adequate insertion. Typically, the fundal wall should be at least $10 \mathrm{~mm}$. The use of a forceps of 18 or $19 \mathrm{~cm}$ length, Allis or Pozzi forceps, is recommended, as this facilitates insertion and helps in assuring anchoring in the fundal midline which is typically the thickest region. Clinical experience shows that access to the uterus and straightening of the utero-cervical axis are facilitated by the lithotomy position for all IUD/IUS insertions. Following insertion, the anchor point should always be visualized and the S-A distance should be measured. If the anchoring appears not to be adequate, removal of the LNG-IUS can be performed immediately with a new insertion being performed in the same session or later. A recent publication provides further details on how to become proficient with insertion of frameless IUDs. ${ }^{30}$ Strictly following the instructions issued by the manufacturer has reduced the perforation rate to $\sim 1 / 1,000$ insertions, which is not higher than the rate observed with framed IUD/IUS. In addition, expulsion rates in the early stages can be nearly eliminated.

FibroPlant will function well if it is well inserted. The force to remove the frameless LNG-IUS, which is greater than the force that is required to remove a conventional copper T-shaped IUD, indicates that spontaneous expulsion of the frameless LNG-IUS is unlikely. ${ }^{31}$ Absence of expulsion is an important advantage of the frameless system. The two expulsions that occurred in the contraceptive study were probably caused by unfamiliarity with the insertion technique. Failure to properly anchor the LNG-IUS in the fundus will usually result in expulsion of the frameless device within weeks of the "insertion" attempt. This contrasts with expulsions occurring with conventional IUDs, which is mainly due to spatial incompatibility with a too small or a too large uterine cavity. The uterus is capable of generating up to $50 \mathrm{~N}$ of myometrial force depending on internal pressure and surface area. If the IUD is not fully expelled, embedment of the frame and/or secondary perforation of the IUD may occur. ${ }^{32}$ The imbalance between the size of the IUD and that of the uterine cavity can result in the production of asymmetrical uterine forces, which can increase patient discomfort, especially while menstruating. Total expulsion of a conventional framed IUD occurs in 5\% to $10 \%$ of women during the first year of use, with $1 \%$ to $2 \%$ per year thereafter. A recent study reported expulsion rates of 8.4 per 100 women at 36 months with the Mirena LNG-IUS in nulliparous women, but 18.8 expulsions per 100 women were observed in adolescents aged 14 to 19 years. ${ }^{33}$ It is likely that many of the IUDs that are not expelled will embed, resulting in patient discomfort, bleeding, and even advance to perforations. Malposition of IUDs occurs frequently and the IUDs are often found displaced in the lower uterine segment and the cervix. ${ }^{34}$ The usual practice is to remove these IUDs. Although full expulsions are serious when they do occur, malpositioning and partial embedment likely have greater impact on patient comfort and thus continuation rates.

Pain is a common side effect of current framed T-shaped IUDs, leading to early removal and poor continuation rates. Although 12 FibroPlant LNG-IUSs were removed because of pelvic pain in the contraceptive study, further questioning of the patients revealed that these removals were unrelated to the frameless LNG-IUS. This is similar with the results obtained with the frameless GyneFix IUD where it was found that only a small percentage of women requested removal for 
this reason. ${ }^{23}$ As it consists of a flexible fiber, it is unlikely that it could cause any pain complaints. In contrast, one can conclude that a woman who complains of cramping pain for days after insertion of a framed IUD has clearly been fitted with an IUD that is not suitable for her uterine cavity. Disproportion between the size of the IUD and the woman's uterine cavity leads to embedment, displacement, partial or total expulsion of the IUD, unintended pregnancy (due to downward displacement, partial, or total expulsion), and abnormal or heavy uterine bleeding. ${ }^{35}$ FibroPlant was developed to improve the relationship with the uterine cavity of parous and nulliparous women to reduce the side effects and expulsion rates of conventional IUDs and increase continuation of use. The experience with the frameless LNG-IUS in young nulliparous and adolescent women with primary dysmenorrhea suggests that the frameless design may be preferred over a framed LNG-IUS, as the absence of a frame is particularly advantageous in these women. ${ }^{28}$ Providers should be aware of the great disparity in size and shape of uterine cavities with at least $50 \%$ of nulliparous women having maximal uterine fundal widths of below $24 \mathrm{~mm}$, with some below $10 \mathrm{~mm} .{ }^{36}$ The use of conventional, framed LNG-IUS may, therefore, not be the best choice for these women.

In addition to the advantageous design features of the frameless LNG-IUS, of significant importance are both the high contraceptive efficacy and high effectiveness in managing menstrual bleeding in women with dysfunctional uterine bleeding, as observed in the clinical trials reported in this paper. The results obtained in the MBL studies show a significant reduction in MBL in heavy and normal menstrual bleeders and subsequent improvement in their serum ferritin levels, occurring soon after treatment initiation. Women harboring intramural or subserous uterine fibroids may also benefit from the use of a LNG-IUS. Adenomyosis-related HMB may also respond to intrauterine treatment with LNG-IUS. ${ }^{37}$

One important disadvantage of LNG-IUS use, well known to gynecologists, is their tendency to cause intermenstrual spotting, which is annoying for women. Counseling following insertion is therefore important and woman's patience will usually be rewarded after a few weeks. To minimize this risk, it appears important that the LNG-IUS be inserted soon after the onset of menstruation.

\section{Conclusion}

The intrauterine administration of low-dose LNG for prolonged periods has significant therapeutic benefits to women with a variety of gynecological conditions. Drug delivery systems capable of both high effectiveness as well as patient tolerability are critical factors allowing for optimal therapy. FibroPlant LNG-IUS is unique as it is frameless, which offers substantial advantages as a frameless design will, by definition, be better tolerated than a framed intrauterine system. Health care providers should realize that up to $40 \%$ of uterine cavities are less than $20 \mathrm{~mm}$ wide and therefore cannot accommodate and tolerate IUDs that are much wider. Hence, it is necessary to assess the uterine cavity size. ${ }^{38}$ Many women suffer from ill-fitting IUDs and one in two women will not use the IUD for the full approved lifespan of the IUD. ${ }^{23}$ Most side effects occurring with framed IUD/IUS can be avoided with a frameless IUD; the risk of displacement and expulsion is low, pain complaints after insertion are usually not present, bleeding, and spotting caused by IUD/ cavity disproportion is, due to its optimal design characteristics, improbable. These are the most common reasons for early removal of a conventional framed IUD/IUS. However, further studies, including comparative trials, are required to substantiate the current findings.

\section{Disclosure}

Dirk Wildemeersch, $\mathrm{MD}, \mathrm{PhD}$, has conducted research in the field of nonhormonal and hormonal, framed and frameless IUDs for 30 years. He assisted in the development of the FibroPlant ${ }^{\circledR}$ LNG-IUS. He did not receive any financial compensation of any kind. The author alone is responsible for the content and writing of this paper. The other authors report no conflicts of interest in this work.

\section{References}

1. Gibson JW, Dunn RL, Perkins BH, Scommegna A. Development of a fibrous IUD delivery system for estradiol/progesterone. In: Zatuchni GI, Goldsmith A, Sciarra JJ, editors. Intrauterine Contraception: Advances and Future Prospects. Philadelphia, PA: Harper and Row; 1985:215-227. (PARFR Series on Fertility Regulation).

2. Segal SJ. The development of Norplant implants. Stud Fam Plann. 1983;14:159-163

3. Luukkainen T, Allonen H, Haukkamaa M, Lähteenmäki P, Nilsson CG, Toivonen J. Five years' experience with levonorgestrel-releasing IUDs. Contraception. 1986;33(2):139-148.

4. Edlund M, Magnuson C, von Schoultz B. Quality of life - a Swedish survey of 2200 women. In: Smith SK, editor. Dysfunctional Uterine Bleeding. London: Royal Society of Medicine Press; 1994.

5. Coulter A, Bradlow J, Agass M. Outcomes of referrals to gynaecology outpatient clinics for menstrual problems: an audit of general practice records. Br J Obstet Gynaecol. 1991;98:789-796.

6. Clarke A, Black N, Rowe P, Mott S, Howie K. Indications for and outcomes of hysterectomy for benign disease: a prospective cohort study. Br J Obstet Gynaecol. 1995;102:611-620.

7. Ryan GL, Syrop CH, Van Voorhis BJ. Role, epidemiology, and natural history of benign uterine mass lesions. Clin Obstet Gynecol. 2005;48: $312-324$

8. JanssenEB, Rijkers ACM,HoppenbrouwersK, MeulemanC,D'HoogheTM. Prevalence of endometriosis diagnosed by laparoscopy in adolescents with dysmenorrhea or chronic pelvic pain: a systematic review. Hum Reprod Update. 2013;19:1570-1582. 
9. Bergeron C, Amant F, Ferenczy A. Pathology and physiopathology of adenomyosis. Best Pract Res Clin Obstet Gynaecol. 2006;20:511-521.

10. Kurman RJ, Kaminski PF, Norris HJ. The behaviour of endometrial hyperplasia. A long-term study of "untreated" hyperplasia in 170 patients. Cancer. 1985;56:403-412.

11. Brown J, Farquhar C. An overview of treatments for endometriosis. JAMA. 2015;313:296-297.

12. Koga K, Takamura M, Fujii T, Osuga Y. Prevention of the recurrence of symptom and lesions after conservative surgery for endometriosis. Fert Steril. 2015;104:793-801.

13. World Health Organization (WHO). Medical Eligibility Criteria for Contraceptive Use. Geneva, Switzerland: WHO; 2000.

14. R Development Core Team. R: A Language and Environment for Statistical Computing. Vienna, Austria: R Foundation for Statistical Computing; 2013. ISBN: 3-900051-07-0. Available from: http:// www.R-project.org. Accessed November 16, 2016.

15. Tietze C, Lewit S. Recommended procedures for the statistical evaluation of intrauterine contraception. Stud Fam Plann. 1972;4:35-42.

16. Farley TMM. Life-table methods for contraceptive research. Stat Med. 1986;5:475-489.

17. Janssen CA, Scholten PC, Heintz APM. A simple visual assessment technique to discriminate between menorrhagia and normal menstrual blood loss. Obstet Gynecol. 1995;85:977-982.

18. Newton J, Barnard G, Collins W. A rapid method for measuring menstrual blood loss using automatic extraction. Contraception. 1977;16: 269-282.

19. Andrade ATL, Souza JP, Shaw ST Jr, Belsey EM, Rowe PJ. Menstrual blood loss and body iron stores in Brazilian women. Contraception. 1991;43:241-249.

20. Hendrickson MR, Kempson RL. Surgical pathology of the uterine corpus. In: Major Problems in Pathology. Vol 12. Philadelphia: WB Saunders; 1980:99-158.

21. Kurman RJ, editor. Blaustein's Pathology of the Female Genital Tract. 4th ed. New York: Springer Verlag; 1995. ISBN 3-540-94166-5.

22. Wildemeersch D, Andrade A. Review of clinical experience with the frameless LNG-IUS for contraception and treatment of heavy menstrual bleeding. Gynecol Endocrinol. 2010;26:383-389.

23. Wildemeersch D, Jandi S, Pett A, Nolte K, Hasskamp T, Vrijens M. Use of frameless intrauterine devices and systems in young nulliparous and adolescent women: results of a multicenter study. Int J Women's Health. 2014;6:727-734.

24. Wildemeersch D, Schacht E. Treatment of menorrhagia with a novel frameless' intrauterine levonorgestrel-releasing drug delivery system: a pilot study. Eur J Contracept Reprod Health Care. 2001;6:93-101.

25. Andrade A, Wildemeersch D. Menstrual blood loss in women using the frameless FibroPlant ${ }^{\mathbb{B}}$ LNG-IUS. Contraception. 2009;79:134-138.
26. Wildemeersch D, Janssens D, Schacht E, Pylyser K, De Wever N. Intrauterine levonorgestrel delivered by a frameless system, combined with systemic estrogeb: acceptability and endometrial safety after 3 years of use in peri- and postmenopausal women. Gynecol Endocrinol. $2005 ; 20: 336-342$.

27. Wildemeersch D, Pylyser K, De Wever N, Dhont M. Management of patients with non atypical and atypical endometrial hyperplasia with a levonorgestrel-releasing intrauterine system: long-term follow-up. Maturitas. 2007;57:210-213.

28. Wildemeersch D, Jandi S, Pett A, Hasskamp T. Management of primary dysmenorrhea in young women with LNG-IUS. J Contraception. 2014;5:23-28.

29. Silverberg SG, Haukkamaa M, Arko H, Nilsson CG, Luukkainen T. Endometrial morphology during long-term use of levonorgestrel-releasing intrauterine devices. Int J Gynecol Pathol. 1986;5:235-241.

30. Wildemeersch D, Hasskamp T. How to become proficient with insertion of frameless IUDs. Obstet Gynecol Int J. 2016;4:2-9.

31. Wildemeersch D. The force required to remove the frameless 0 -suture anchoring system: comparison between pre- and postmenopausal women. Contraception. 2004;69:513-515.

32. Goldstuck N, Hasskamp T, Jandi S, Pett A, Wildemeersch D. Geometric foundations of intrauterine device complications and implications for IUD uses - importance of IUD size to maximize tolerability and prevent early discontinuation. J Reproduktionsmed Endokrinol Online. 2015;12:255-259. German.

33. Madden T, McNicholas C, Zhao Q, et al. Association of age and parity with intrauterine device expulsion. Obstet Gynecol. 2014;124: 718-726.

34. Braaten KP, Benson CB, Maurer R, et al. Malpositioned intrauterine contraceptive devices: risk factors, outcomes, and future pregnancies. Obstet Gynecol. 2011;118:1014-1020.

35. Goldstuck N, Wildemeersch D. Role of uterine forces in intrauterine device embedment, perforation, and expulsion. Int J Womens Health. 2014; 6:735-744.

36. Nolte K, Jandi S, Julen O, Hasskamp T, Wildemeersch D. A multicenter study assessing uterine cavity width in over 150 nulliparous women with IUD or seeking IUD insertion using 3D sonography. Clin Obstet Gynecol Reprod Med. 2016;2:193-198.

37. Bragheto AM, Caserta N, Bahamondes L, Petta CA. Effectiveness of the levonorgestrel-releasing intrauterine system in the treatment of adenomyosis diagnosed and monitored by magnetic resonance imaging. Contraception. 2007;76:195-199.

38. Wildemeersch D, Hasskamp T, Goldstuck N. Side effects of intrauterine devices are often related to disproportion with the endometrial cavity - is there a role for preinsertion ultrasound? Eur J Obstet Gynecol Reprod Biol. 2016;201:215-218.
International Journal of Women's Health

\section{Publish your work in this journal}

The International Journal of Women's Health is an international, peerreviewed open-access journal publishing original research, reports, editorials, reviews and commentaries on all aspects of women's healthcare including gynecology, obstetrics, and breast cancer. The manuscript management system is completely online and includes

\section{Dovepress}

a very quick and fair peer-review system, which is all easy to use Visit http://www.dovepress.com/testimonials.php to read real quotes from published authors. 